\title{
A possible link between BDNF and mTOR in control of food intake
}

\author{
Nobuyuki Takei ${ }^{1}{ }^{*}, K_{a z u o ~ F u r u k a w a}{ }^{1,2}$, Osamu Hanyu ${ }^{2}$, Hirohito Sone ${ }^{2}$ and Hiroyuki Nawa ${ }^{1}$ \\ ' Department of Molecular Neurobiology, Brain Research Institute, Niigata University, Niigata, Japan \\ ${ }^{2}$ Department of Hematology, Endocrinology and Metabolism, Faculty of Medicine, Niigata University, Niigata, Japan
}

\author{
Edited by: \\ Tanya Zilberter, Infotonic Conseil, \\ France \\ Reviewed by: \\ Marcelo Febo, University of Florida, \\ USA \\ Yuri Zilberter, INSERM-UMR 1106 \\ Institut de Neurosciences des \\ Systèmes, France \\ *Correspondence: \\ Nobuyuki Takei, Department of \\ Molecular Neurobiology, Brain \\ Research Institute, Niigata University, \\ 1 Asahimachi, Niigata 951-8585, \\ Japan \\ e-mail:nobtak@bri.niigata-u.ac.jp
}

\begin{abstract}
Food intake is intricately regulated by glucose, amino acids, hormones, neuropeptides, and trophic factors through a neural circuit in the hypothalamus. Brain-derived neurotrophic factor (BDNF), the most prominent neurotrophic factor in the brain, regulates differentiation, maturation, and synaptic plasticity throughout life. Among its many roles, BDNF exerts an anorexigenic function in the brain. However, the intracellular signaling induced by BDNF to control food intake is not fully understood. One candidate for the molecule involved in transducing the anorexigenic activity of BDNF is the mammalian target of rapamycin (mTOR). mTOR senses extracellular amino acids, glucose, growth factors, and neurotransmitters, and regulates anabolic reactions response to these signals. Activated mTOR increases protein and lipid synthesis and inhibits protein degradation. In the hypothalamus, mTOR activation is thought to reduce food intake. Here we summarize recent findings regarding BDNF- and mTOR-mediated feeding control, and propose a link between these molecules in eating behavior.
\end{abstract}

\section{Keywords: BDNF, mTOR, mTORC1, food intake regulation, body weight, AMPK, nutrients}

\section{INTRODUCTION}

Several lines of evidence indicate that neurons in the hypothalamus sense nutrient sufficiency. These neurons are regulated by many factors involved in feeding control, such as leptin (Morton etal., 2006). The roles of many other feedingrelated peptides, both orexigenic and anorexigenic, have also been extensively studied. For example, clear obesity and leanness phenotypes are observed in knockout mice lacking pro-opiomelanocortin (POMC; a precursor of $\alpha$-melanocytestimulating hormone (MSH); Yaswen et al., 1999) and melaninconcentrating hormone (MCH; Shimada etal., 1998), respectively. Recently, another factor involved in regulation of feeding and metabolic regulation in the brain has come into the spotlight: brain-derived neurotrophic factor (BDNF; Ooi et al., 2012; Rios, 2013; Vanevski and Xu, 2013; Marosi and Mattson, 2014).

\section{BRAIN-DERIVED NEUROTROPHIC FACTOR}

Brain-derived neurotrophic factor is the most prominent neurotrophic factor in the central nervous system. Indeed, BDNF and its cognate high-affinity receptor, TrkB, are widely expressed in the brain from development to adulthood. BDNF promotes differentiation, maturation, and survival of neurons, and plays important roles in synaptic plasticity through the activation of TrkB, a receptor tyrosine kinase (Nawa and Takei, 2001; Park and Poo, 2013). TrkB-expressing (i.e., BDNF-responsive) neurons are distributed in the arcuate nucleus (ARC), paraventricular nucleus (PVN), lateral hypothalamus ( $\mathrm{LH})$, ventromedial nucleus (VMH), and dorsomedial nucleus (DMN) of the hypothalamus (Yan etal., 1997).

\section{BDNF AND REGULATION OF FOOD INTAKE}

The first evidence that BDNF is involved in body weight control came from a rather serendipitous result. While assessing the neuroprotective effects of neurotrophins, Lapchak and Hefti (1992) found that chronic intracerebroventricular (ICV) infusion of BDNF in adult rats after fimbrial lesion reduced body weight. Subsequent systematic experiments also revealed that ICV injection of BDNF suppressed appetite and promoted weight loss in rats (Pelleymounter et al., 1995). The second clear line of evidence that BDNF plays crucial role in food intake comes from studies of genetically manipulated mice. Mice heterozygously deleted for the gene encoding $\operatorname{BDNF}\left(B d n f^{+-}\right)$produce half of the wild-type level of BDNF protein and exhibit a severely obese phenotype due to overeating (Lyons et al., 1999; Kernie et al., 2000). Furthermore, brain-specific deletion of Bdnf (Rios etal., 2001), deletion of dendritic BDNF mRNA (Liao et al., 2012), shRNA-mediated knockdown of BDNF using a viral vector (Unger et al., 2007), and a hypomorphic allele of Trkb that expresses only a quarter of $\operatorname{TrkB}$ all result in hyperphagia, obesity, and metabolic imbalances such as hyperglycemia (Xu et al., 2003).

Genotype-phenotype interactions indicate that BDNF-TrkB signals also play important roles in weight control in humans. For instance, a de novo missense mutation of the TRKB gene, Tyr722Cys, which leads to a defect in downstream signaling, was identified in an 8-year-old male who presented with hyperphagia, severe obesity, and developmental delay (Yeo et al., 2004). Similarly, patients with Wilms' tumor, aniridia, genitourinary anomalies, and mental retardation (WAGR) syndrome, who have a truncation of chromosome 11, exhibit hyperphagia and obesity. Analysis of the genomes of WAGR patients revealed that 
they are heterozygous for deletion of BDNF. Patients with $B D N F$ haploinsufficiency were all obese, whereas only $20 \%$ of WAGR patients without BDNF deletion were obese (Han et al., 2008). A common single-nucleotide polymorphism (SNP) of BDNF, G196A, produces the amino acid substitution Val66Met in the prodomain. The Val66Met mutant exhibits defects in intracellular trafficking and activity-dependent release of mature BDNF (Egan et al., 2003). A genome-wide association study linked this SNP to susceptibility to obesity in humans (Beckers et al., 2008; Speliotes et al., 2010; Waterhouse and Xu, 2013). Likewise, in an experimental model, G196A knock-in mice exhibit increased body weight (Chen et al., 2006). Because these mutations in humans are all genomic, not somatic, the effect of BDNF deficiency on metabolic abnormalities may arise from systemic and/or developmental activities. Indeed, it has been proposed that BDNF contributes to metabolism in peripheral organs (Nakagawa et al., 2000; Hanyu et al., 2003). However, brain- or hypothalamus-specific deletion or knockdown of BDNF induces overeating and obesity. These results suggest that BDNF acts directly on the hypothalamic circuit that regulates food intake and metabolism, thereby controling body weight. In addition, BDNF injection into the brain can rescue the obese phenotype of mutant mice. These results indicate that BDNF exerts its anorexic action in an acute, temporally specific manner, and that the effects of loss of BDNF on feeding behavior are not the result of developmental defects in neural circuits. BDNF also acts on midbrain dopaminergic neurons, which are involved in hedonic eating related to the reward/addiction system (Cordeira et al., 2010; Alsiö et al., 2012). Thus, this system may contribute to overeating in individuals carrying these mutations. Future studies should investigate these issues in greater detail.

Previous studies of eating behavior have focused on extracellular cues and neural circuits in the hypothalamus, but have not looked as closely at intracellular signaling mechanisms. Considering the acute effect of BDNF on food intake, we wondered what signaling molecules play major roles in BDNF-mediated feeding control. Mammalian target of rapamycin (mTOR), a kinase that governs metabolism in peripheral cells, has attracted attention as a regulator of food intake through the brain (Cota, 2009; Wiczer and Thomas, 2010; Howell and Manning, 2011; André and Cota, 2012). BDNF is a major activator of mTOR in neurons (Takei etal., 2001, 2004; Inamura et al., 2005) therefore, we hypothesize that the anorexigenic action of BDNF is mediated by mTOR in neurons. Before elaborating on this idea, we will provide a brief introduction of $\mathrm{mTOR}$ and its signaling pathways.

\section{MAMMALIAN TARGET OF RAPAMYCIN}

Mammalian target of rapamycin is the mammalian ortholog of yeast TOR, which is the target molecule of rapamycin, an anti-fungal and immunosuppressant compound. mTOR is a serine/threonine kinase that forms two complexes, mTOR complex 1 (mTORC1) and 2, which have different molecular partners. mTORC1 is a key component of the nutrient-sensing network that controls cellular metabolism: it integrates various extracellular cues, such as nutrients (amino acids and glucose) and growth factors, and it regulates various biochemical processes, including translation, autophagy, transcription, and lipid biosynthesis. These biochemical reactions induce anabolic states and thereby promote cell growth. The signaling pathways upstream and downstream of mTORC1 have recently been elucidated (Laplante and Sabatini, 2012; Takei and Nawa, 2014); we have provided a simplified schematic of neuronal mTOR signaling in Figure 1 Leucine, taken up by the system L-amino acid transporter, activates mTORC1 (Ishizuka et al., 2008). Growth factors such as BDNF (Takei et al., 2001, 2004; Inamura et al., 2005), insulin (Lee et al., 2005), and insulin-like growth factor (Quevedo et al., 2002) activate the phosphoinositide 3-kinase (PI3K)/Akt pathway through their tyrosine kinase receptors in neurons. Akt directly phosphorylates tuberous sclerosis complex 2 (TSC2), a suppressor of Rheb that activates mTORC1. When glucose levels are sufficient, AMP-activated protein kinase (AMPK) activity decreases, and thus mTORC1 becomes active (Dash et al., 2006). These inputs converge on mTORC1; therefore, the availability of amino acids and/or glucose is essential for growth factor-mediated mTORC1 activation (Hara etal., 1998; Ishizuka etal., 2013). mTORC1 phosphorylates eukaryotic initiation factor 4E-binding protein (4EBP) and thereby stimulates translation. In addition, phosphorylation of p70S6 kinase (p70S6K) by mTORC1 also promotes translation and lipid biosynthesis, whereas phosphorylation of ULK1 inhibits autophagy. All of these processes increase total protein and lipid levels in the cell and thereby increase cellular mass (Laplante and Sabatini, 2012; Takei and Nawa, 2014). It should be noted that mTORC1 signaling is regulated by a feedback mechanism: mTORC1 and p70S6K phosphorylate

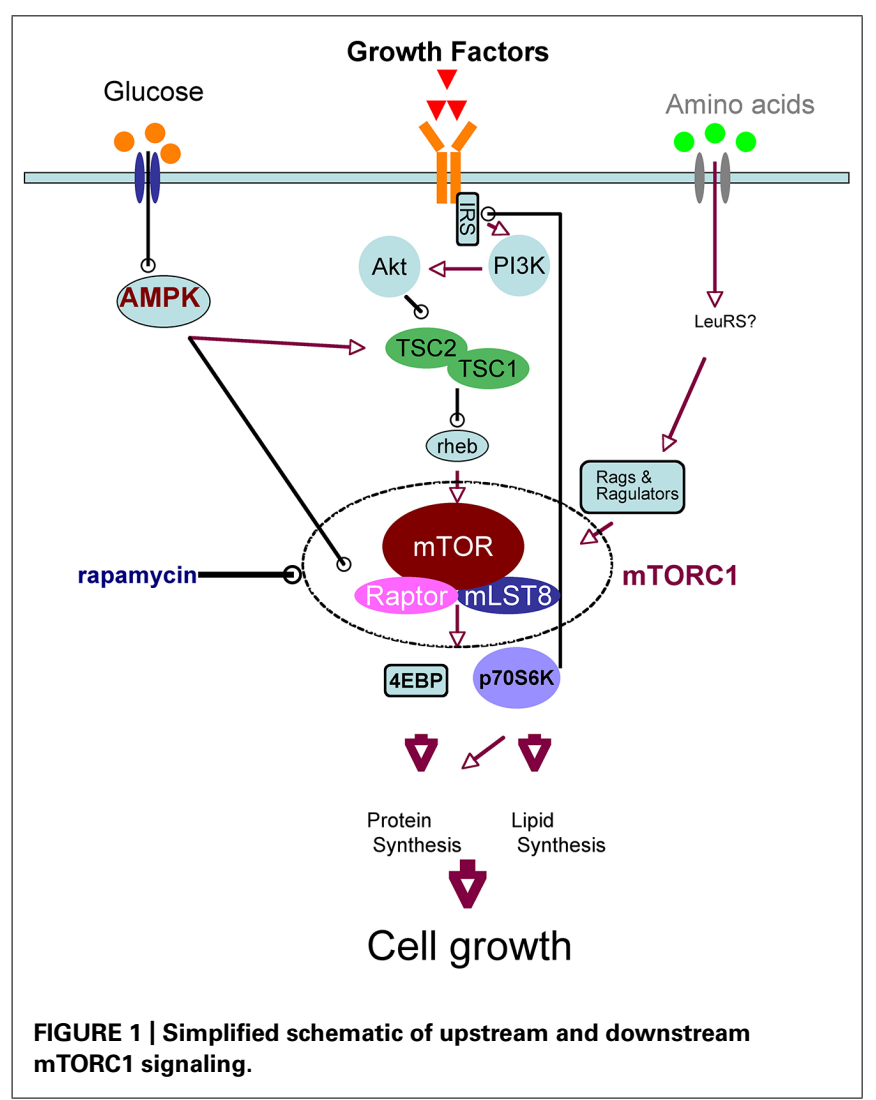


and inactivate insulin receptor substrate (IRS; Tremblay and Marette, 2001; Figure 1), which interacts receptor tyrosine kinases such as insulin receptor and TrkB (Yamada et al., 1997). Thus, prolonged activation or overactivation of mTORC1 results in desensitization of this signaling cascade; this is thought to be one mechanism of insulin resistance (Tremblay and Marette, 2001).

\section{mTOR AND REGULATION OF FOOD INTAKE}

In the brain, the first hint of a relationship between food intake and mTORC1 signaling was provided by the involvement of AMPK on feeding. AMPK is a cellular fuel gage that senses the AMP/ATP ratio and turns off metabolic pathways that consume ATP (Kemp et al., 2003). Because AMPK itself plays a key role in metabolism, the regulation of hypothalamic AMPK was initially investigated independently of mTOR (Minokoshi et al., 2004, 2008). Leptin, insulin (Minokoshi et al., 2004), and cilliary neurotrophic factor (CNTF; Steinberg et al., 2006) reduce AMPK activity in the hypothalamus similarly to re-feeding after fasting; thus, these molecules suppress food intake and thereby reduce body weight. By contrast, adiponectin (Kubota et al., 2007), ghrelin, and AICAR (Andersson et al., 2004), a pharmacological activator of AMPK, stimulate AMPK activity, and thus increase food intake and body weight. Moreover, adenovirus-mediated expression of dominantnegative (DN) AMPK reduces food intake and body weight, whereas expression of constitutive-active (CA) AMPK increases them (Minokoshi et al., 2004). Recent findings regarding the signaling network (Figure 1) suggest that regulation of food intake by AMPK may converge on the mTORC1 system in the hypothalamus. AMPK activates TSC2, a suppressor of mTORC1, and thereby inhibits mTORC1 (Inoki et al., 2003). In addition, AMPK phosphorylates Raptor, a scaffold protein of mTORC1, also leading to inhibition of mTORC1 (Gwinn et al., 2008). Thus, like rapamycin, AMPK activation suppresses mTORC1 and increases food intake.

Direct evidence that mTORC1 signaling might be coupled with feeding has been reported (Cota et al., 2006). Similar to the action of leptin, ICV injection of leucine activates mTORC1 and reduces food intake. Moreover, the effects of both leucine and leptin in the brain on mTORC1 signaling and food intake are counteracted by rapamycin, a specific mTORC1 inhibitor. Fasting and re-feeding also affect mTORC1 signaling in neurons in the hypothalamus. It remains unclear which area of the hypothalamus, as well as which types of neurons, participates in mTORC1-mediated regulation of food intake. The answer to this question might be provided by precise analysis using methods such as immunohistochemistry with phospho-specific antibodies to mTOR network molecules. Studies of mTORC1 signaling on food intake using genetically modified mice have yielded seemingly paradoxical consequences. Global deletion of p70S6K (s6 $\mathrm{k}^{-/-}$), a downstream signaling molecule of mTORC1, protects against diet-induced obesity (Um et al., 2004). By contrast, injection of an adenovirus vector carrying DN-p70S6K into the mediobasal hypothalamus increases food intake and body weight, whereas overexpression of CA p70S6K reduces both parameters (Blouet et al., 2008; Ono et al., 2008). Furthermore, conditional deletion of $T s c 1$, an upstream suppressor of mTORC1 in hypothalamic neurons (and in beta cells of the pancreas), induces hyperphagic obesity, and hypothalamic POMC neuronspecific deletion of $T s c 1$ results in the same phenotype (Mori et al., 2009). Because Tsc1 deletion induces mTORC1 activation, this phenomenon seems contradictory to the anorexic effect of mTORC1.

Two issues complicate our understanding of mTORC1's action on food intake and weight control: the negative-feedback mechanism and the difference between the peripheral (or systemic) and central (brain) activities of mTORC1. Prolonged activation of mTORC1 signaling induced by gene knockouts of upstream or downstream molecules may cause inactivation of mTORC1 in the hypothalamus via negative-feedback. Thus, for example, knockout of $T s c 1$ may cause orexigenic rather than anorexigenic effects due to long-lasting feedback suppression of mTORC1. Precise biochemical analysis of mTORC1 signaling in these animals may help to resolve this paradox. Systemic mTORC1 pathway activation induces cell growth in many organs, and thereby increases body weight; this may explain why global knockout of p70S6K suppresses weight gain (Blouet et al., 2008; Ono et al., 2008). Nevertheless, because the organism must maintain

\section{Cell \\ nutrients Growth factors (e.g. BDNF)
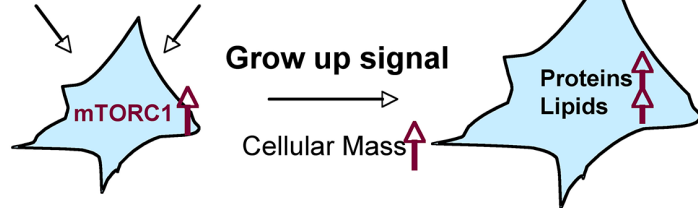 \\ Organism}

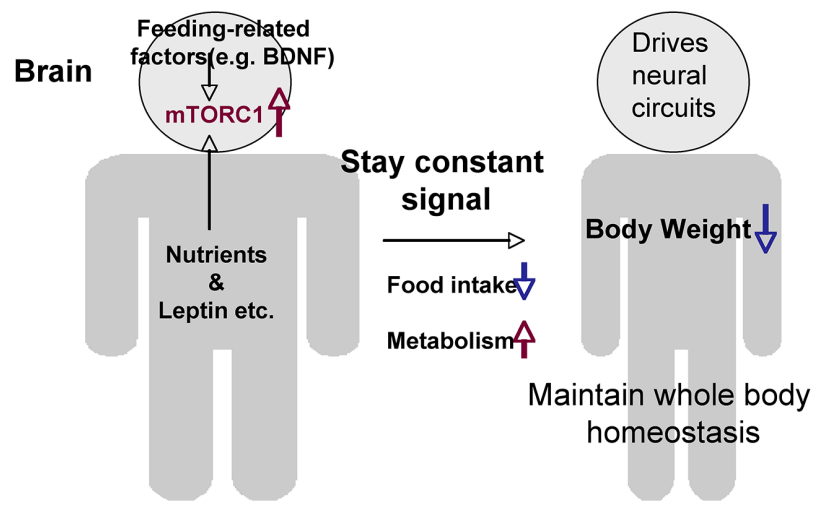

FIGURE 2 | Hypothetical roles of mTORC1 on metabolism and food intake, comparing the cellular and organismal levels. Through the activation of $\mathrm{mTORC} 1$, cells that receive nutrients and growth factors grow by increasing total levels of proteins and lipids. Cells (neurons) in the brain activate $\mathrm{mTORC} 1$ in response to nutrients and a variety of feeding-related factors. The resultant signals give the order to stop eating and maintain whole-body homeostasis. 
whole-body homeostasis, it is quite likely that phasic activation of mTORC1 in hypothalamic neurons drives an anorexic state. In other words, mTORC1 senses the "satiety" signal in the brain in order to maintain an appropriate body weight (Figure 2).

\section{BDNF AND mTOR}

Amino acids, glucose, and growth factors induce mTORC1 activation in the hypothalamus and reduce food intake. In addition to leucine and leptin, CNTF (Cota et al., 2008) and bone morphogenic protein 9 (BMP9; Townsend et al., 2012) also suppress food intake. Culture studies have revealed that these molecules elicit mTORC1 signaling in neuronal cells. Leucine activates mTORC1 through the system L-amino acid transporter in primary cultured neurons (Ishizuka et al., 2008), whereas CNTF induces the phosphorylation of STAT3 via mTORC1 in neuroblastoma cells (Yokogami et al., 2000). These phenomena are similar to the effects of BDNF on both mTORC1 signaling and food intake. On the cellular level, BDNF is a potent activator of mTORC1 in neurons, and it stimulates anabolic responses such as protein synthesis (Takei et al., 2001, 2004). Although there is as yet no direct evidence, it is very likely that the anorexigenic action of BDNF is mediated by mTORC1 activation in the hypothalamus. Importantly, BDNF-mediated mTORC1 activation is limited by glucose availability (Ishizuka et al., 2013). Because glucose and amino acids are indispensable for the maintenance of homeostasis, this observation suggests the existence of a safeguard system in which glucose sufficiency overrides other mTORC1activating stimuli in neurons. Therefore, it is necessary to obtain direct evidence that the anorexigenic action of BDNF is really mediated by mTORC1 signaling, either via the use of mTOR inhibitors such as rapamycin or knockdown of mTORC1 components in the hypothalamus. In addition, it is also important to determine which types of neurons in the hypothalamus are actually responsible for mTORC1-mediated feeding control. The use of a unique promoter-driven Cre-mouse (such as POMC-Cre) to make conditional knockout in mTOR or mTORC1 components in certain hypothalamic neurons may be useful in this regard.

In unicellular organisms and at the single-cell level in metazoans, nutrient uptake and subsequent mTORC1 activation lead to cell growth (i.e., increase in cellular mass and/or proliferation). In multicellular organisms, the brain regulates food intake to maintain whole-body homeostasis. Thus, mTORC1 in hypothalamic neurons senses many complex signals, both from the periphery (e.g., glucose, amino acids, insulin, leptin, and ghrelin) and from neural networks within the brain (e.g., via peptides and BDNF; Figure 2). The mechanisms by which the brain controls feeding behavior are complex, but mTORC1 may represent a cellular crossroads for the regulation of food intake and metabolism by nutrients and other inputs.

\section{ACKNOWLEDGMENTS}

This study was supported by the Grant-in-Aid for Scientific Research C (JSPS) and the Grant-in-Aid for Scientific Research on Innovative Areas (MEXT).

\section{REFERENCES}

Alsiö, J., Olszewski, P. K., Levine, A. S., and Schiöth, H. B. (2012). Feedforward mechanisms: addiction-like behavioral and molecular adaptations in overeating. Front. Neuroendocrinol. 33:127-139. doi: 10.1016/j.yfrne.2012. 01.002

Andersson, U., Filipsson, K., Abbott, C. R., Woods, A., Smith, K., Bloom, S. R., et al. (2004). AMP-activated protein kinase plays a role in the control of food intake. J. Biol. Chem. 279, 12005-12008. doi: 10.1074/jbc.C3005 57200

André, C., and Cota, D. (2012). Coupling nutrient sensing to metabolic homoeostasis: the role of the mammalian target of rapamycin complex 1 pathway. Proc. Nutr. Soc. 71, 502-510. doi: 10.1017/S0029665112000754

Beckers, S., Peeters, A., Zegers, D., Mertens, I., Van Gaal, L., and Van Hul, W. (2008). Association of the BDNF Val66Met variation with obesity in women. Mol. Genet. Metab. 95, 110-112. doi: 10.1016/j.ymgme.2008.06.008

Blouet, C., Ono, H., and Schwartz, G. J. (2008). Mediobasal hypothalamic p70 S6 kinase 1 modulates the control of energy homeostasis. Cell Metab. 8, 459-467. doi: 10.1016/j.cmet.2008.10.004

Chen, Z. Y., Jing, D., Bath, K. G., Ieraci, A., Khan, T., Siao, C. J., et al. (2006). Genetic variant BDNF (Val66Met) polymorphism alters anxiety-related behavior. Science 314, 140-143. doi: 10.1126/science.1129663

Cordeira, J. W., Frank, L., Sena-Esteves, M., Pothos, E. N., and Rios, M. (2010). Brain-derived neurotrophic factor regulates hedonic feeding by acting on the mesolimbic dopamine system. J. Neurosci. 30, 2533-2541. doi: 10.1523/JNEUROSCI.5768-09.2010

Cota, D. (2009). Mammalian target of rapamycin complex 1 (mTORC1) signaling in energy balance and obesity. Physiol. Behav. 97, 520-524. doi: 10.1016/ j.physbeh.2009.03.006

Cota, D., Matter, E. K., Woods, S. C., and Seeley, R. J. (2008). The role of hypothalamic mammalian target of rapamycin complex 1 signaling in dietinduced obesity. J. Neurosci. 28, 7202-7208. doi: 10.1523/JNEUROSCI.138908.2008

Cota, D., Proulx, K., Smith, K. A., Kozma, S. C., Thomas, G., Woods, S. C., et al. (2006). Hypothalamic mTOR signaling regulates food intake. Science 312, 927930. doi: 10.1126/science.1124147

Dash, P. K., Orsi, S. A., and Moore, A. N. (2006). Spatial memory formation and memory-enhancing effect of glucose involves activation of the tuberous sclerosis complex-Mammalian target of rapamycin pathway. J. Neurosci. 26, 8048-8056. doi: 10.1523/JNEUROSCI.0671-06.2006

Egan, M. F., Kojima, M., Callicott, J. H., Goldberg, T. E., Kolachana, B. S., Bertolino, A., et al. (2003). The BDNF val66met polymorphism affects activity-dependent secretion of BDNF and human memory and hippocampal function. Cell 112, 257-269. doi: 10.1016/S0092-8674(03)00035-7

Gwinn, D. M., Shackelford, D. B., Egan, D. F., Mihaylova, M. M., Mery, A., Vasquez, D. S., et al. (2008). AMPK phosphorylation of raptor mediates a metabolic checkpoint. Mol. Cell 30, 214-226. doi: 10.1016/j.molcel.2008. 03.003

Han, J. C., Liu, Q. R., Jones, M., Levinn, R. L., Menzie, C. M., JeffersonGeorge, K. S.,et al. (2008). Brain-derived neurotrophic factor and obesity in the WAGR syndrome. N. Engl. J. Med. 359, 918-927. doi: 10.1056/NEJMoa 0801119

Hanyu, O., Yamatani, K., Ikarashi, T., Soda, S., Maruyama, S., Kamimura, T., et al. (2003). Brain-derived neurotrophic factor modulates glucagon secretion from pancreatic alpha cells: its contribution to glucose metabolism. Diabetes Obes. Metab. 5, 27-37. doi: 10.1046/j.1463-1326.2003.00238.x

Hara, K., Yonezawa, K., Weng, Q. P., Kozlowski, M. T., Belham, C., and Avruch, J. (1998). Amino acid sufficiency and mTOR regulate p70S6 kinase and eIF4E BP1 through a common efector mechanism. J. Biol. Chem. 273, 14484-14494. doi: $10.1074 /$ jbc.273.23.14484

Howell, J. J., and Manning, B. D. (2011). mTOR couples cellular nutrient sensing to organismal metabolic homeostasis. Trends Endocrinol. Metab. 22, 94-102. doi: 10.1016/j.tem.2010.12.003

Inamura, N., Nawa, H., and Takei, N. (2005). Enhancement of translation elongation in neurons by brain-derived neurotrophic factor: implications for mammalian target of rapamycin signaling. J. Neurochem. 95, 1438-1445. doi: 10.1111/j.14714159.2005.03466.x

Inoki, K., Zhu, T., and Guan, K. L. (2003). TSC2 mediates cellular energy response to control cell growth and survival. Cell 115, 577-590. doi: 10.1016/S00928674(03)00929-2 
Ishizuka, Y., Kakiya, N., Nawa, H., and Takei, N. (2008). Leucine induces phosphorylation and activation of p70S6K in cortical neurons via the system $\mathrm{L}$ amino acid transporter. J. Neurochem. 106, 934-942. doi: 10.1111/j.1471-4159.2008.05438.x

Ishizuka, Y., Kakiya, N., Witters, L. A., Oshiro, N., Shirao, T., and Nawa, H. (2013). AMP-activated protein kinase counteracts brain-derived neurotrophic factor-induced mammalian target of rapamycin complex 1 signaling in neurons. J. Neurochem. 127, 66-77. doi: 10.1111/jnc.12362

Kemp, B. E., Stapleton, D., Campbell, D. J., Chen, Z. P., Murthy, S., Walter, M., et al. (2003). AMP-activated protein kinase, super metabolic regulator. Biochem. Soc. Trans. 31(Pt 1), 162-168.

Kernie, S. G., Liebl, D. J., and Parada, L. F. (2000). BDNF regulates eating behavior and locomotor activity in mice. EMBO J. 19, 1290-1300. doi: 10.1093/emboj/19.6.1290

Kubota, N., Yano, W., Kubota, T., Yamauchi, T., Itoh, S., Kumagai, H., et al. (2007). Adiponectin stimulates AMP-activated protein kinase in the hypothalamus and increases food intake. Cell Metab. 6, 55-68. doi: 10.1016/j.cmet.2007. 06.003

Lapchak, P. A., and Hefti, F. (1992). BDNF and NGF treatment in lesioned rats: effects on cholinergic function and weight gain. Neuroreport 3, 405-408. doi: 10.1097/00001756-199205000-00007

Laplante, M., and Sabatini, D. M. (2012). mTOR signaling in growth control and disease. Cell 149, 274-293. doi: 10.1016/j.cell.2012.03.017

Lee, C. C., Huang, C. C., Wu, M. Y., and Hsu, K. S. (2005). Insulin stimulates postsynaptic density-95 protein translation via the phosphoinositide 3-kinaseAkt-mammalian target of rapamycin signaling pathway. J. Biol. Chem. 280 18543-18550. doi: 10.1074/jbc.M414112200

Liao, G. Y., An, J. J., Gharami, K., Waterhouse, E. G., Vanevski, F., Jones, K. R., et al (2012). Dendritically targeted Bdnf mRNA is essential for energy balance and response to leptin. Nat. Med. 18, 564-571. doi: 10.1038/nm.2687

Lyons, W. E., Mamounas, L. A., Ricaurte, G. A., Coppola, V., Reid, S. W., Bora, S. H., et al. (1999). Brain-derived neurotrophic factor-deficient mice develop aggressiveness and hyperphagia in conjunction with brain serotonergic abnormalities. Proc. Natl. Acad. Sci. U.S.A. 96, 15239-15244. doi: 10.1073/pnas.96.26. 15239

Marosi, K., and Mattson, M. P. (2014). BDNF mediates adaptive brain and body responses to energetic challenges. Trends Endocrinol. Metab. 25, 89-98. doi: 10.1016/j.tem.2013.10.006

Minokoshi, Y., Alquier, T., Furukawa, N., Kim, Y. B., Lee, A., Xue, B., et al. (2004). AMP-kinase regulates food intake by responding to hormonal and nutrient signals in the hypothalamus. Nature 428, 569-574. doi: 10.1038/nature 02440

Minokoshi, Y., Shiuchi, T., Lee, S., Suzuki, A., and Okamoto, S. (2008). Role of hypothalamic AMP-kinase in food intake regulation. Nutrition 24, 786-790. doi: 10.1016/j.nut.2008.06.002

Mori, H., Inoki, K., Münzberg, H., Opland, D., Faouzi, M., Villanueva, E. C., et al. (2009). Critical role for hypothalamic mTOR activity in energy balance. Cell Metab. 9, 362-374. doi: 10.1016/j.cmet.2009.03.005

Morton, G. J., Cummings, D. E., Baskin, D. G., Barsh, G. S., and Schwartz, M. W. (2006). Central nervous system control of food intake and body weight. Nature 443, 289-295. doi: 10.1038/nature05026

Nakagawa, T., Tsuchida, A., Itakura, Y., Nonomura, T., Ono, M., Hirota, F., et al. (2000). Brain-derived neurotrophic factor regulates glucose metabolism by modulating energy balance in diabetic mice. Diabetes 49, 436-444. doi: 10.2337/diabetes.49.3.436

Nawa, H., and Takei, N. (2001). BDNF as an anterophin; a novel neurotrophic relationship between brain neurons. Trends Neurosci. 24, 683-684. doi: 10.1016/S0166-2236(00)01955-X

Ono, H., Pocai, A., Wang, Y., Sakoda, H., Asano, T., Backer, J. M., et al. (2008). Activation of hypothalamic S6 kinase mediates diet-induced hepatic insulin resistance in rats. J. Clin. Invest. 118, 2959-2968. doi: 10.1172/JCI34277

Ooi, C. L., Kennedy, J. L., and Levitan, R. D. (2012). A putative model of overeating and obesity based on brain-derived neurotrophic factor: direct and indirect effects. Behav. Neurosci. 126, 505-514. doi: 10.1037/ a0028600

Park, H., and Poo, M. M. (2013). Neurotrophin regulation of neural circuit development and function. Nat. Rev. Neurosci. 14, 7-23. doi: 10.1038/ nrn3379
Pelleymounter, M. A., Cullen, M. J., and Wellman, C. L. (1995). Characteristics of BDNF-induced weight loss. Exp. Neurol. 131, 229-238. doi: 10.1016/00144886(95)90045-4

Quevedo, C., Salinas, M., and Alcázar, A. (2002). Regulation of cap-dependent translation by insulin-like growth factor-1 in neuronal cells. Biochem. Biophys. Res. Commun. 291, 560-566. doi: 10.1006/bbrc.2002.6479

Rios, M. (2013). BDNF and the central control of feeding: accidental bystander or essential player? Trends Neurosci. 36, 83-90. doi: 10.1016/j.tins.2012. 12.009

Rios, M., Fan, G., Fekete, C., Kelly, J., Bates, B., Kuehn, R., et al. (2001) Conditional deletion of brain-derived neurotrophic factor in the postnatal brain leads to obesity and hyperactivity. Mol. Endocrinol. 15, 1748-1757. doi: 10.1210/mend.15.10.0706

Shimada, M., Tritos, N. A., Lowell, B. B., Flier, J. S., and Maratos-Flier, E. (1998). Mice lacking melanin-concentrating hormone are hypophagic and lean. Nature 396, 670-674. doi: 10.1038/25341

Speliotes, E. K.,Willer, C. J., Berndt, S. I., Monda, K. L., Thorleifsson, G., Jackson, A. U., et al. (2010). Association analyses of 249,796 individuals reveal 18 new loci associated with body mass index. Nat. Genet. 42, 937-948. doi: 10.1038/ ng.686

Steinberg, G. R., Watt, M. J., Fam, B. C., Proietto, J., Andrikopoulos, S., Allen, A. M., et al. (2006). Ciliary neurotrophic factor suppresses hypothalamic AMPkinase signaling in leptin-resistant obese mice. Endocrinology 147, 3906-3914. doi: 10.1210/en.2005-1587

Takei, N., Inamura, N., Kawamura, M., Namba, H., Hara, K., Yonezawa, K., et al. (2004). Brain-derived neurotrophic factor induces mammalian target of rapamycin-dependent local activation of translation machinery and protein synthesis in neuronal dendrites. J. Neurosci. 24, 9760-9769. doi: 10.1523/JNEUROSCI.1427-04.2004

Takei, N., Kawamura, M., Hara, K., Yonezawa, K., and Nawa, H. (2001). Brainderived neurotrophic factor enhances neuronal translation by activating multiple initiation processes: comparison with the effects of insulin. J. Biol. Chem. 276, 42818-42825. doi: 10.1074/jbc.M103237200

Takei, N., and Nawa, H. (2014). mTOR signaling and its roles in normal and abnormal brain development. Front. Mol. Neurosci. 7:28. doi: $10.3389 /$ fnmol.2014.00028

Townsend, K. L., Suzuki, R., Huang, T. L., Jing, E., Schulz, T. J., Lee, K., et al. (2012). Bone morphogenetic protein 7 (BMP7) reverses obesity and regulates appetite through a central mTOR pathway. FASEB J. 26, 2187-2196. doi: 10.1096/fj.11199067

Tremblay, F., and Marette, A. (2001). Amino acid and insulin signaling via the mTOR/p70 S6 kinase pathway. A negativefeedback mechanism leading to insulin resistance in skeletal muscle cells. J. Biol. Chem. 276, 3805238060 .

Um, S. H., Frigerio, F., Watanabe, M., Picard, F., Joaquin, M., Sticker, M., et al. (2004). Absence of S6K1 protects against age- and diet-induced obesity while enhancing insulin sensitivity. Nature 431, 200-205. doi: 10.1038/nature 02866

Unger, T. J., Calderon, G. A., Bradley, L. C., Sena-Esteves, M., and Rios, M. (2007). Selective deletion of Bdnf in the ventromedial and dorsomedial hypothalamus of adult mice results in hyperphagic behavior and obesity. J. Neurosci. 27, 1426514274. doi: 10.1523/JNEUROSCI.3308-07.2007

Vanevski, F., and Xu, B. (2013). Molecular and neural bases underlying roles of BDNF in the control of body weight. Front. Neurosci. 7:37. doi: 10.3389/fnins.2013.00037

Waterhouse, E. G., and Xu, B. (2013). The skinny on brain-derived neurotrophic factor: evidence from animal models to GWAS. J. Mol. Med. (Berl.) 91, 1241-1247. doi: 10.1007/s00109-013-1071-8

Wiczer, B. M., and Thomas, G. (2010). The role of the mTOR pathway in regulating food intake. Curr. Opin. Drug Discov. Dev. 13, 604-612.

Xu, B., Goulding, E. H., Zang, K., Cepoi, D., Cone, R. D., Jones, K. R., et al. (2003). Brain-derived neurotrophic factor regulates energy balance downstream of melanocortin-4 receptor. Nat. Neurosci. 6, 736-742. doi: 10.1038/ nn 1073

Yamada, M., Ohnishi, H., Sano, S. I., Nakatani, A., Ikeuchi, T., and Hatanaka, H. (1997). Insulin receptor substrate (IRS)-1 and IRS2 are tyrosine-phosphorylated and associated with phosphatidylinositol 3-kinase in response to brain-derived 
neurotrophic factor in cultured cerebral cortical neurons. J. Biol. Chem. 272, 30334-30339. doi: 10.1074/jbc.272.48.30334

Yan, Q., Radeke, M. J., Matheson, C. R., Talvenheimo, J., Welcher, A. A., and Feinstein, S. C. (1997). Immunocytochemical localization of TrkB in the central nervous system of the adult rat. J. Comp. Neurol. 378, 135-157. Erratum in: J. Comp. Neurol. 382, 546-547. doi: 10.1002/(SICI) 1096-9861(19970203)378:1<135::AIDCNE8 $>3.0 . \mathrm{CO} ; 2-5$

Yaswen, L., Diehl, N., Brennan, M. B., and Hochgeschwender, U. (1999). Obesity in the mouse model of pro-opiomelanocortin deficiency responds to peripheral melanocortin. Nat. Med. 5, 1066-1070. doi: 10.1038/12506

Yeo, G. S., Connie Hung, C. C., Rochford, J., Keogh, J., Gray, J., Sivaramakrishnan, S., et al. (2004). A de novo mutation affecting human TrkB associated with severe obesity and developmental delay. Nat. Neurosci. 7, 1187-1189. doi: $10.1038 / \mathrm{nn} 1336$

Yokogami, K., Wakisaka, S., Avruch, J., and Reeves, S. A. (2000). Serine phosphorylation and maximal activation of STAT3 during CNTF signaling is mediated by the rapamycin target mTOR. Curr. Biol. 10, 47-50. doi: 10.1016/S0960-9822(99)00268-7
Conflict of Interest Statement: The authors declare that the research was conducted in the absence of any commercial or financial relationships that could be construed as a potential conflict of interest.

Received: 27 June 2014; accepted: 09 September 2014; published online: 25 September 2014.

Citation: Takei N, Furukawa K, Hanyu O, Sone H and Nawa H (2014) A possible link between BDNF and mTOR in control of food intake. Front. Psychol. 5:1093. doi: 10.3389/fpsyg.2014.01093

This article was submitted to Eating Behavior, a section of the journal Frontiers in Psychology.

Copyright (c) 2014 Takei, Furukawa, Hanyu, Sone and Nawa. This is an openaccess article distributed under the terms of the Creative Commons Attribution License (CC BY). The use, distribution or reproduction in other forums is permitted, provided the original author(s) or licensor are credited and that the original publication in this journal is cited, in accordance with accepted academic practice. No use, distribution or reproduction is permitted which does not comply with these terms. 